\title{
$\mathrm{AlCl}_{3}$ 催化 3-(2-氨基乙基)吡咯的合成
}

\author{
白 冰*,a 徐芳琳 ${ }^{a}$ 杨 静 $a$ 张改红 ${ }^{a}$ 毛多斌 $a$ 王宁*,b \\ ( ${ }^{a}$ 郑州轻工业大学 食品与生物工程学院 郑州 450000) \\ ( $b$ 河南卷烟工业烟草薄片有限公司 河南许昌 461000)
}

\begin{abstract}
摘要 3-(2-氨基乙基)吡咯是形成 6-氮杂吲哚骨架的重要中间体，由于吡咯 2 位为高活性位点，难以直接通过傅克反应 合成 3-(2-氨基乙基)吡咯. 报道了在吡咯 $\alpha$ 位引入拉电子基团, 形成对角 $\beta$ 位的定位效应, 以无水 $\mathrm{AlCl}_{3}$ 为催化剂, 通过
\end{abstract} 傅克反应在吡咯 3 位加成硝基烯烃, 进而得到取代的 3-(2-氨基乙基)吡咯.

关键词 三氯化铝; 3-(2-氨基乙基)吡咯; 6-氮杂吲哚

\section{Synthesis of 3-(2-Aminoethyl)pyrroles Catalyzed by $\mathrm{AlCl}_{3}$}

\author{
Bai, Bing ${ }^{*, a} \quad$ Xu, Fanglin ${ }^{a} \quad$ Yang, Jing $^{a} \quad$ Zhang, Gaihong $^{a}$ \\ Mao, Duobin ${ }^{a} \quad$ Wang, Ning*,b
}

( ${ }^{a}$ School of Food \& Biological Engineering, Zhengzhou University of Light Industry, Zhengzhou 450000)

( ${ }^{b}$ Henan Cigarette Industrial Reconstituted Tobacco Sheet Co., Ltd., Xuchang, Henan 461000)

\begin{abstract}
Aminoethyl)pyrroles are important intermediates for the formation of the 6-azaindole skeleton. It was difficult to synthesize 3-(2-aminoethyl)pyrroles from pyrrole by Friedel-Crafts alkylation directly because of the inactive site. The electron withdrawing groups were introduced at the $\alpha$-position of pyrrole to form a positioning effect at the diagonal $\beta$-position. With anhydrous $\mathrm{AlCl}_{3}$ as the catalyst, nitroalkenes could be added to the 3-position of pyrrole via Friedel-Crafts alkylation to obtain substituted 3-(2-aminoethyl)pyrroles.

Keywords aluminium chloride; 3-(2-aminoethyl)pyrrole; 6-azaindole
\end{abstract}

氮杂吲哚类化合物 ${ }^{[1]}$ 包括各种取代的 4-氮杂吲 哚 ${ }^{[1]} 、 5$-氮杂吲哚 ${ }^{[2]} 、 6$-氮杂吲哚[3]和 7-氮杂吲哚 ${ }^{[4]}$, 因 具有广泛的生物活性，成为近年来研究的热点 ${ }^{[5]}$. 6-氮 杂吲哚是氮杂吲哚家族重要的一员, 具有潜在的抗肿 瘤 ${ }^{[6]}$ 、治疗糖尿病 ${ }^{[7]}$ 及抗艾滋病毒 ${ }^{[8]}$ 药物开发的价值(图 1). 传统的 6-氮杂吲哚合成法有 Fischer 法 ${ }^{[9]}$, Bartoli 环 化法 ${ }^{[10]}$ 和 Diels-Alder 法 ${ }^{[1]}$, 这几种方法底物结构有较 大局限性, 得到的氮杂吲哚化合物结构单一, 缺乏可修 饰性, 因此, 如何高效便捷地构建 6-氮杂吲哚骨架仍是 需要持续关注的问题.

3-(2-氨基乙基)吡咯类化合物作为中间体，可以与 醛或酮通过 pictect-spengler 反应形成 6-氮杂吲哚骨 架 ${ }^{[2]}$, 且由于其可以和各种醛酮类化合物缩合, 因此可 以得到结构类型多样的 6-氮杂吲哚类化合物. 目前有关 3 -(2-氨基乙基)吡咯的合成报道较少 ${ }^{[13]}$, 尽管 $\beta$-硝基烯<smiles>CCN1CCN(C(=O)Nc2cc(-c3cn(C4CCOCC4)c4cnccc34)ccn2)CC1</smiles><smiles>COc1cnc(-n2cnc(C)n2)c2c1c(C(=O)C(=O)N1CCN(C(=O)c3ccccc3)CC1)cn2COP(=O)(O)O</smiles>

GNF2133, DYRK1A, IC $50=6 \mathrm{nmol} / \mathrm{L} \quad \mathrm{HIV}-1(\mathrm{LAl}) \mathrm{EC}_{50}=0.4 \mathrm{nmol} / \mathrm{L}$<smiles>COc1cc(C2=C(c3c[nH]c4cnccc34)C(=O)NC2=O)cc(OC)c1OC</smiles>

VEGFR-2 IC ${ }_{50}=48 \mathrm{nmol} / \mathrm{L}$

FLT-3 $\quad I C_{50}=18 \mathrm{nmol} / \mathrm{L}$ GSK-3 $3 \quad I_{50}=9 \mathrm{nmol} / \mathrm{L}$

图 1 6-氮杂吲哚类活性化合物

Figure 1 Bioactive compounds featuring 6-azaindole system

\footnotetext{
* Corresponding author. E-mail: baibing82@163.com; ningbotao2006@163.com Received February 1, 2021; revised March 2, 2021; published online March 19, 2021.

Project supported by the Department of Science and Technology of Henan Province (No. 192102110212). 河南省科技厅(No. 192102110212)资助项目.
} 
烃与吡咯通过傅克烷基化反应，可获得硝基取代的吡 咯, 进而通过还原可得到氨基乙基吡咯, 然而受 $\mathrm{N}$ 电负 性影响, 吡咯 2 位为高活性位点, 与硝基烯烃进行的傅 克反应优先得到 2-取代吡咯[14]. 利用大位阻硅烷化试剂 对吡咯 $\mathrm{N}$ 进行保护可以降低其 2 位的活性, 但在获得 3取代产物的同时, 仍有相当比例的产物为 2-取代吡 咯 ${ }^{[13 \mathrm{~b}]}$. Meshram 等 ${ }^{[15]}$ 曾报道利用 $\mathrm{Zn}(\mathrm{OAc})_{2}$ 作为催化剂 催化吡咯与硝基烯烃的反应, 可得到 3-取代吡咯, 但我 们重复实验所得到的产物实际上仍为 2-取代吡咯, 且 Lancianesi 等 ${ }^{[13 b]}$ 的报道也证实了这一点. 由此可见, 在 吡咯低活性的 3 位位点进行傅克烷基化反应, 进而合成 3-(2-氨基乙基)吡咯仍是一个挑战性课题. 本工作通过 在吡咯 $\alpha$ 位引入拉电子基团, 形成对角 $\beta$ 位的定位效应 的策略 ${ }^{[16]}$, 以无水 $\mathrm{AlCl}_{3}$ 为催化剂, 选择性地在吡咯 3 位加成硝基苯乙烯, 通过该方法可得到一系列取代的 3-(2-氨基乙基)吡咯.

\section{1 结果与讨论}

\section{1 合成路线设计}

3-(2-氨基乙基)吡咯的合成路线如 Scheme 1 所示. 吡咯 $\alpha$ 位电子云密度远大于 $\beta$ 位, 要想在 $\beta$ 位发生烷基 化或酰基化反应, 必须要降低 $\alpha$ 位电子云密度. 我们先 是在 $\alpha$ 位引入三氯乙酰基, 利用定位效应在对角 $\beta$ 位与 硝基苯乙烯亲电加成, 意外的是未得到硝基取代的加成 产物 3-(2-硝基乙基)吡咯, 而是生成了肜, 由于其还原 比较困难, 随后我们将 $\alpha$-三氯乙酰基吡咯(1)通过醇钠 转变为 2-吡咯甲酸甲酯(2)之后, 肟 3 的还原能够顺利进 行. 化合物 3 的加成位点可由 ${ }^{1} \mathrm{H}^{-}{ }^{1} \mathrm{H}$ COSY 谱图确认.

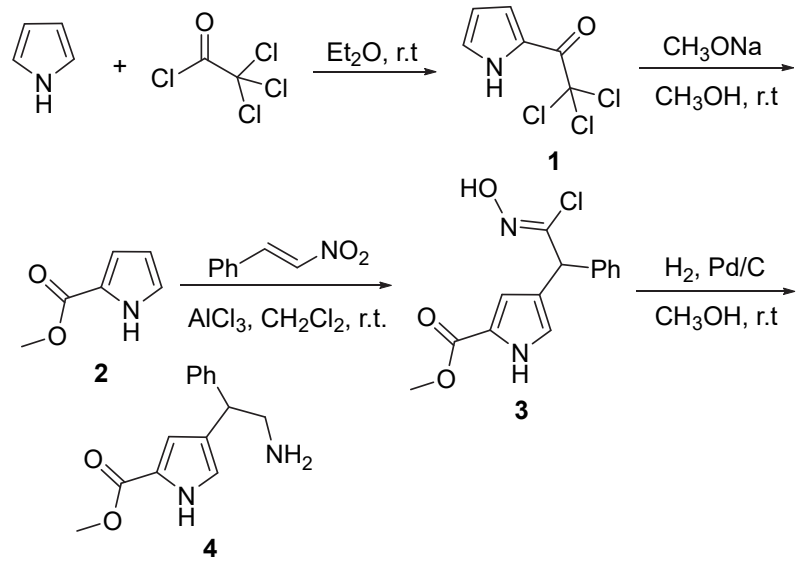

图式 13 -(2-氨基乙基)吡咯合成路线

Scheme 1 Synthetic routes of 3-(2-aminoethyl)pyrroles

\section{2 反应条件篮选}

首先对傅克反应催化剂进行了篮选, 结果见表 1 . 由于吡咯 3 位活性较弱, 在利用三氟甲磺酸钇 $\left[\mathrm{Y}\left(\mathrm{CF}_{3}-\right.\right.$
$\left.\left.\mathrm{SO}_{3}\right)_{3}\right]$ 与三氟甲磺酸镧 $\left[\mathrm{La}\left(\mathrm{CF}_{3} \mathrm{SO}_{3}\right)_{3}\right]$ 作为催化剂时, 未 得到目标产物(Entries 1，2)，同时也未得到 3-(2-硝基乙 基)吡咯; 尽管 $\mathrm{Zn}(\mathrm{OAc})_{2}$-乙醇体系可以以温和方式获得 吡咯 2 位烷基化产物, 但无法催化吡咯 3 位的烷基化反 应(Entry 3); 其它路易斯酸如 $\mathrm{FeCl}_{3}, \mathrm{TiCl}_{4}$ 和三氟化硼乙 醚，曾被用来成功催化吡咯的酰基化反应，然而对吡咯 3 位的烷基化并不适用(Entries $4 \sim 6$ ); 无水 $\mathrm{AlCl}_{3}$ 和 $\mathrm{EtAlCl}_{2}$ 在室温下均可催化吡咯 3 位的烷基化反应 (Entries 7,8), 其中 $\mathrm{AlCl}_{3}$ 在用量为 2 equiv.时, 能够以 $79 \%$ 的产率获得化合物 3 , 具有较好的催化效果; 以二 氯乙烷为溶剂时, 产物产率有所降低(Entry 9); 以苯为 溶剂时, 目标产物生成量极少(Entry 10), 同时硝基苯乙 烯被消耗，说明硝基苯乙烯优先与苯与发生反应.

表 1 催化剂的优化 ${ }^{a}$

Table 1 Optimization of catalysts

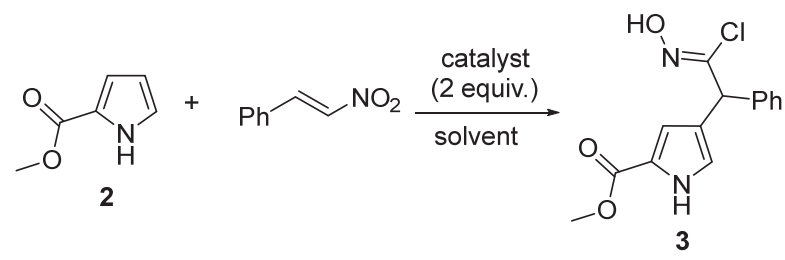

\begin{tabular}{cllc}
\hline Entry & \multicolumn{1}{c}{ Catalyst } & Solvent & Yield $^{b} \%$ \\
\hline 1 & $\mathrm{Y}\left(\mathrm{CF}_{3} \mathrm{SO}_{3}\right)_{3}$ & $\mathrm{CH}_{2} \mathrm{Cl}_{2}$ & 0 \\
2 & $\mathrm{La}\left(\mathrm{CF}_{3} \mathrm{SO}_{3}\right)_{3}$ & $\mathrm{CH}_{2} \mathrm{Cl}_{2}$ & 0 \\
3 & $\mathrm{Zn}(\mathrm{OAc})_{2}$ & $\mathrm{EtOH}$ & 0 \\
4 & $\mathrm{FeCl}_{3}$ & $\mathrm{CH}_{2} \mathrm{Cl}_{2}$ & 0 \\
5 & $\mathrm{TiCl}_{4}$ & $\mathrm{CH}_{2} \mathrm{Cl}_{2}$ & 0 \\
6 & $\mathrm{BF}_{3} \cdot \mathrm{Et}_{2} \mathrm{O}$ & $\mathrm{CH}_{2} \mathrm{Cl}_{2}$ & 0 \\
7 & $\mathrm{EtAlCl}_{2}$ & $\mathrm{CH}_{2} \mathrm{Cl}_{2}$ & 65 \\
8 & $\mathrm{AlCl}_{3}$ & $\mathrm{CH}_{2} \mathrm{Cl}_{2}$ & 79 \\
9 & $\mathrm{AlCl}_{3}$ & $\mathrm{C}_{2} \mathrm{H}_{4} \mathrm{Cl}_{2}$ & 67 \\
10 & $\mathrm{AlCl}_{3}$ & $\mathrm{Benzene}$ & Trace \\
11 & $\mathrm{AlCl}_{3}$ & $\mathrm{PhCl}^{2}$ & 30 \\
\hline
\end{tabular}

${ }^{a}$ Reaction condition: 2 ( $\left.2 \mathrm{mmol}\right), \beta$-nitrostyrene $(2 \mathrm{mmol})$, catalyst $(4 \mathrm{mmol})$, solvent $(10 \mathrm{~mL})$, r.t. ${ }^{b}$ Isolated yield.

随后对 $\mathrm{AlCl}_{3}$ 催化硝基苯乙烯对 $\mathbf{2}$ 的加成反应进行 了优化, 主要考察了催化剂用量及反应温度的影响, 结 果见表 2. 当 $\mathrm{AlCl}_{3}$ 用量为 1 equiv.时，以中等产率得到 肜 3 (Entry 1); 随着催化剂用量增加, 产物产率逐渐提 高, 至催化剂用量为 3 equiv.时, 产率达到最高的 $87 \%$ (Entry 3), 此后基本稳定. 对不同反应温度考察发现， 随着反应温度的降低, 肜 3 的产率下降幅度较大, 至 $-40{ }^{\circ} \mathrm{C}$ 时, 没有产物生成. $\mathrm{Tu}$ 等 ${ }^{[14 a]}$ 曾报道了利用 $\mathrm{AlCl}_{3}$ 催化硝基苯乙烯对芳环的加成反应，当温度控制在室温 时, 产物为重排结构的肜, 而当反应温度为 $-78{ }^{\circ} \mathrm{C}$ 时, 产物为硝基烷烃. 而硝基苯乙烯对 2-吡咯甲酸甲酯 $\mathbf{2}$ 的 加成反应，在低温下并未得到任何加成产物，可能是因 为 2 的活性较低, 难以在低温下形成中间体所致; 当反 
应温度升高至 $35{ }^{\circ} \mathrm{C}$, 产率未见提高(Entries 9, 10). 因 此, 反应条件确定为: $\mathrm{AlCl}_{3}$ 用量为 3 equiv., 以 $\mathrm{CH}_{2} \mathrm{Cl}_{2}$ 为溶剂在室温下反应.

表 2 反应条件优化 ${ }^{a}$

Table 2 Optimization of reaction conditions

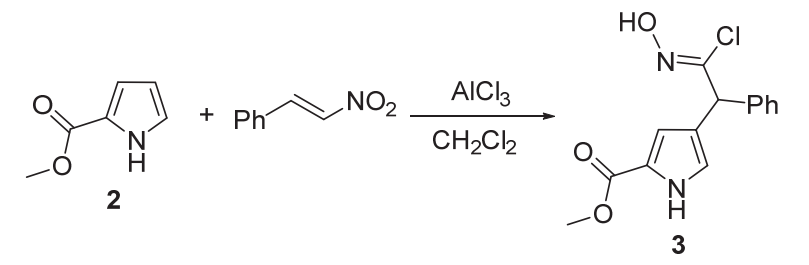

\begin{tabular}{ccccc}
\hline Entry & $\mathrm{AlCl}_{3} /$ equiv. & Temp. $/{ }^{\circ} \mathrm{C}$ & Time/h & Yield $^{b} \%$ \\
\hline 1 & 1.0 & r.t & 16 & 57 \\
2 & 2.0 & r.t & 16 & 79 \\
3 & 3.0 & r.t & 10 & 87 \\
4 & 4.0 & r.t & 10 & 82 \\
5 & 3.0 & 0 & 24 & 62 \\
6 & 3.0 & -20 & 24 & 15 \\
7 & 3.0 & -40 & 24 & 0 \\
8 & 3.0 & -78 & 24 & 0 \\
9 & 3.0 & 35 & 10 & 86 \\
10 & 2.0 & 35 & 16 & 80
\end{tabular}

${ }^{a}$ Reaction condition: 2 (2 mmol), $\beta$-nitrostyrene $(2 \mathrm{mmol})$, solvent $(10 \mathrm{~mL})$. ${ }^{b}$ Isolated yield.

\section{3 反应底物适用范围}

为了考察反应的应用范围, 根据上述选定的催化条 件, 对底物的适用性进行了拓展, 结果如表 3 所示. 在 无水 $\mathrm{AlCl}_{3}$ 催化下, 苯环上具有不同取代基的硝基苯乙 烯均可与 2-吡咯甲酸甲酯发生反应, 包括卤素取代、甲
基取代和甲氧基取代，大部分反应产率在 $80 \%$ 以上. 其 中以 3,4-二甲氧基取代的硝基苯乙烯为底物时(Entry 9), 产率较低, 为 $61 \%$, 这可能与取代基有关. 化合物 3 通 过还原可得到 3-(2-氨基乙基)吡咯 4, 其中非卤素取代 的肟可通过钯碳加氢还原以较高产率得到目标产物; 卤 素取代的肜在钯碳体系下容易脱去卤素, 采用 $\mathrm{NaBH}_{4} / \mathrm{NiCl}_{2}$ 还原体系可避免卤素的脱除.

\section{2 结论}

从吡咯出发, 通过在其 $\alpha$ 位引入拉电子基团, 形成 对角 $\beta$ 位的定位效应, 以无水 $\mathrm{AlCl}_{3}$ 为催化剂, 可顺利在 吡咯 3 位加成硝基苯乙烯, 进而还原得到取代的 3-(2氨基乙基)吡咯.

\section{3 实验部分}

\section{1 仪器与试剂}

Bruker AVANCE III 600 核磁共振波谱仪; AB Sciex TripleTOF 6600 高分辨质谱仪; Thermo Nicolet 5700 红 外光谱仪; 上海申光 SGW X-4A 熔点仪.

吡咯、三氯乙酰氯、硝基烯烃和钯碳均购自百灵威 (北京)科技有限公司; 三氯化铝购自福晨化工; 其它试 剂均购自国药集团化学试剂有限公司.

\section{2 实验方法}

\subsection{1 三氯乙酰基吡咯(1)的合成}

将三氯乙酰氯(8.56 g, $49.3 \mathrm{mmol})$ 溶于无水 $\mathrm{Et}_{2} \mathrm{O}(20$

表 3 3-(2-氨基乙基)吡咯的合成 ${ }^{a}$

Table 3 Synthesis of 3-(2-aminoethyl)pyrroles

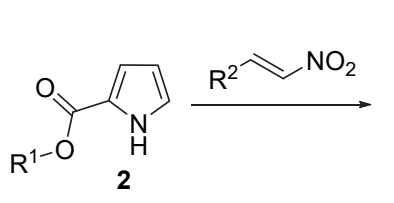<smiles>[R]OC(=O)c1cc(C([R])/C(Cl)=N\O)c[nH]1</smiles><smiles>[R]OC(=O)c1cc(C([R])CN)c[nH]1</smiles>

\begin{tabular}{cllcclc}
\hline Entry & \multicolumn{1}{c}{$\mathrm{R}^{1}$} & \multicolumn{1}{c}{$\mathrm{R}^{2}$} & Time/h & $\mathbf{3}\left(\right.$ Yield $\left.^{b} \%\right)$ & {$[\mathrm{H}]$} & $\mathbf{4}\left(\mathrm{Yield}^{b} / \%\right)$ \\
\hline 1 & $\mathrm{CH}_{3}$ & $\mathrm{Ph}$ & 8 & $\mathbf{3 a}(87)$ & $\mathrm{Pd} / \mathrm{C}, \mathrm{H}_{2}$ & $\mathbf{4 a}(79)$ \\
2 & $\mathrm{CH}_{3}$ & $4-\mathrm{MeC}_{6} \mathrm{H}_{4}$ & 8 & $\mathbf{3 b}(88)$ & $\mathrm{Pd} / \mathrm{C}, \mathrm{H}_{2}$ & $\mathbf{4 b}(80)$ \\
3 & $\mathrm{CH}_{3}$ & $2-\mathrm{FC}_{6} \mathrm{H}_{4}$ & 6 & $\mathbf{3 c}(85)$ & $\mathrm{Pd} / \mathrm{C}, \mathrm{H}_{2}$ & $\mathbf{P d}(82)$ \\
4 & $\mathrm{CH}_{3}$ & $4-\mathrm{FC}_{6} \mathrm{H}_{4}$ & 7 & $\mathbf{3 d}(83)$ & $\mathrm{NaB}, \mathrm{H}_{2}$ & $\mathbf{4 d}(84)$ \\
5 & $\mathrm{CH}_{3}$ & $4-\mathrm{ClC}_{6} \mathrm{H}_{4}$ & 8 & $\mathbf{3 e}(85)$ & $\mathrm{NaBH} \mathrm{Ni}_{2}$ & $\mathbf{4 e}(78)$ \\
6 & $\mathrm{CH}_{3}$ & $4-\mathrm{BrCl}_{6} \mathrm{H}_{4}$ & 7 & $\mathbf{3 f}(87)$ & $\mathrm{Pd} / \mathrm{C}_{2} \mathrm{H}_{2}$ & $\mathbf{4 f}(70)$ \\
7 & $\mathrm{CH}_{3}$ & $4-\mathrm{MeOC}_{6} \mathrm{H}_{4}$ & 8 & $\mathbf{3 g}(88)$ & $\mathrm{NaBH} / \mathrm{NiCl}_{2}$ & $\mathbf{4 g}(85)$ \\
8 & $\mathrm{CH}_{3}$ & $3-\mathrm{BrC}_{6} \mathrm{H}_{4}$ & 6 & $\mathbf{3 h}(80)$ & $\mathrm{Pd} / \mathrm{C}_{2} \mathrm{H}_{2}$ & $\mathbf{4 h}(69)$ \\
9 & $\mathrm{CH}_{3}$ & $3,4-\left(\mathrm{MeO}_{2} \mathrm{C}_{6} \mathrm{H}_{3}\right.$ & 16 & $\mathbf{3 i}(61)^{c}$ & $\mathrm{NaBH} / \mathrm{NiCl}_{2}$ & $\mathbf{4 i}(81)$ \\
10 & $\mathrm{CH}_{3}$ & $2,4-\mathrm{Cl}_{2} \mathrm{C}_{6} \mathrm{H}_{3}$ & 7 & $\mathbf{3 j}(84)$ & $\mathrm{Pd} / \mathrm{C}, \mathrm{H}_{2}$ & $\mathbf{4 j}(79)$ \\
11 & $\mathrm{CH}_{3} \mathrm{CH}_{2}$ & $\mathrm{Ph}_{12}$ & 8 & $\mathbf{3 k}(86)$ & $\mathrm{Pd} / \mathrm{C}, \mathrm{H}_{2}$ & $\mathbf{4 k}(83)$ \\
12 & $\mathrm{CH}_{3} \mathrm{CH}_{2}$ & $3-\mathrm{BrC}_{6} \mathrm{H}_{4}$ & 6 & $\mathbf{3 l}(82)$ & $\mathbf{4 l}(71)$ \\
\hline
\end{tabular}

${ }^{a}$ Reaction conditions: 2 (12 mmol), nitrostyrenes (12 mmol), $\mathrm{AlCl}_{3}(36 \mathrm{mmol})$, solvent $(50 \mathrm{~mL}) ; 3(10 \mathrm{mmol}), \mathrm{Pd} / \mathrm{C}(w=10 \%)$ or $3(4 \mathrm{mmol}), \mathrm{NiCl}_{2} \bullet 6 \mathrm{H}_{2} \mathrm{O}(4 \mathrm{mmol})$, $\mathrm{NaBH}_{4}(48 \mathrm{mmol}){ }^{b}$ Isolated yield. ${ }^{c}$ Uncompleted reaction. 
$\mathrm{mL})$ 中, 冷却至 $10{ }^{\circ} \mathrm{C}$, 缓慢滴加吡咯 $(3 \mathrm{~g}, 44.8 \mathrm{mmol})$, $15 \mathrm{~min}$ 加毕, 升至室温反应 $3 \mathrm{~h}$, 加入 $15 \mathrm{~mL}$ 质量分数为 $1 \%$ 的 $\mathrm{K}_{2} \mathrm{CO}_{3}$ 溶液, 振荡, 分出醚层, 加入 $10 \mathrm{~mL}$ EtOAc 稀释, 活性碳脱色, 硅藻土过滤, 减压蒸干溶剂, 放置 $10 \mathrm{~min}$ 后析出固体, 石油醚重结晶得到白色针晶 $9.5 \mathrm{~g}$, 产率 91\%. m.p. $72 \sim 74{ }^{\circ} \mathrm{C} ;{ }^{1} \mathrm{H}$ NMR $\left(600 \mathrm{MHz}, \mathrm{CDCl}_{3}\right)$ $\delta: 9.48(\mathrm{~s}, 1 \mathrm{H}), 7.39$ (s, 1H), 7.17 (t, $J=2.1 \mathrm{~Hz}, 1 \mathrm{H}), 6.39$ (dt, $J=4.2,2.5 \mathrm{~Hz}, 1 \mathrm{H}) ;{ }^{13} \mathrm{C} \mathrm{NMR}\left(150 \mathrm{MHz}, \mathrm{CDCl}_{3}\right) \delta$ : 173.20, 127.04, 122.98, 121.14, 111.88, 94.92; IR (film) $v$ : 3316, 1649, 1383, 1135, 1034, 806, 684, $593 \mathrm{~cm}^{-1}$; HRMS-ESI calcd for $\mathrm{C}_{6} \mathrm{H}_{5} \mathrm{Cl}_{3} \mathrm{NO}[\mathrm{M}+\mathrm{H}]^{+}$211.9431, found 211.9427.

\section{2 .2 化合物 $\mathbf{2 a} \sim \mathbf{2 b}$ 的合成}

将金属钠 $(2 \mathrm{~g}, 84.7 \mathrm{mmol})$ 溶于无水 $\mathrm{CH}_{3} \mathrm{OH}(50 \mathrm{~mL})$ 中, 加入化合物 1 (6 g, $28.2 \mathrm{mmol})$, 室温反应 $2 \mathrm{~h}$, 蒸除 溶剂, 加入 EtOAc $(30 \mathrm{~mL})$, 饱和 $\mathrm{NaCl}$ 水溶液洗涤, 有 机相无水 $\mathrm{Na}_{2} \mathrm{SO}_{4}$ 干燥，蒸干溶剂得到 2a.

2-吡咯甲酸甲酯(2a): 白色固体, 产率 $89 \%$. m.p. $71 \sim 72{ }^{\circ} \mathrm{C} ;{ }^{1} \mathrm{H}$ NMR $\left(600 \mathrm{MHz}, \mathrm{CDCl}_{3}\right) \delta: 9.32$ (brs, $\left.1 \mathrm{H}\right)$, $6.96(\mathrm{td}, J=2.7,1.5 \mathrm{~Hz}, 1 \mathrm{H}), 6.92$ (ddd, $J=3.8,2.4,1.5$ $\mathrm{Hz}, 1 \mathrm{H}), 6.27(\mathrm{dt}, J=3.6,2.6 \mathrm{~Hz}, 1 \mathrm{H}), 3.86(\mathrm{~s}, 3 \mathrm{H}) ;{ }^{13} \mathrm{C}$ NMR $\left(150 \mathrm{MHz}, \mathrm{CDCl}_{3}\right) \delta: 161.69,122.95,122.63$, 115.28, 110.47, 51.47; IR (film) v: 3283, 2958, 1665, 1442, $1319,1126,752,610 \mathrm{~cm}^{-1}$; HR-MS-ESI calcd for $\mathrm{C}_{6} \mathrm{H}_{8^{-}}$ $\mathrm{NO}_{2}[\mathrm{M}+\mathrm{H}]^{+} 126.0550$, found 126.0552 .

2-吡咯甲酸乙酯 $(\mathbf{2 b})$ : 白色固体, 产率 $92 \%$. m.p. $40 \sim 42{ }^{\circ} \mathrm{C} ;{ }^{1} \mathrm{H}$ NMR $\left(600 \mathrm{MHz}, \mathrm{CDCl}_{3}\right) \delta: 9.43$ (brs, $1 \mathrm{H}$ ), $6.96 \sim 6.95(\mathrm{~m}, 1 \mathrm{H}), 6.93 \sim 6.92(\mathrm{~m}, 1 \mathrm{H}), 6.27 \sim 6.25(\mathrm{~m}$, $1 \mathrm{H}), 4.33(\mathrm{q}, J=7.1 \mathrm{~Hz}, 1 \mathrm{H}), 1.36(\mathrm{t}, J=7.1 \mathrm{~Hz}, 1 \mathrm{H}) ;{ }^{13} \mathrm{C}$ NMR $\left(150 \mathrm{MHz}, \mathrm{CDCl}_{3}\right) \delta: 161.38,122.97,122.85$, 115.15, 110.36, 60.35, 14.46; IR (film) v: 3283, 2959, 1669, 1442, 1315, 1126, 753, $608 \mathrm{~cm}^{-1}$; HR-MS-ESI calcd for $\mathrm{C}_{7} \mathrm{H}_{9} \mathrm{NO}_{2} \mathrm{Na}[\mathrm{M}+\mathrm{Na}]^{+} 162.0525$, found 162.0522 .

\subsection{3 化合物 $\mathbf{3 a} \sim 3 \mathrm{~m}$ 的合成}

无水 $\mathrm{AlCl}_{3}(4.7 \mathrm{~g}, 36 \mathrm{mmol})$ 悬浮于无水 $\mathrm{CH}_{2} \mathrm{Cl}_{2}(50$ $\mathrm{mL})$ 中, 冷至 $0{ }^{\circ} \mathrm{C}$, 加入硝基苯乙烯 $(1.8 \mathrm{~g}, 12 \mathrm{mmol})$, 搅拌 $30 \mathrm{~min}$ 后加入 $2(1.5 \mathrm{~g}, 12 \mathrm{mmol})$, 室温反应 $8 \mathrm{~h}$, 加 入 $10 \mathrm{~mL}$ 水, 分液, 有机相用饱和 $\mathrm{NaCl}$ 水溶液洗涤, 干 燥, 蒸除溶剂, 残留物进行硅胶柱层析, 石油醚/乙酸乙 酯 $(V: V=6: 1)$ 洗脱得到 $3 \mathbf{a}$.

4-(2-氯-2-肜基-1-苯基乙基)-吡咯-2-甲酸甲酯(3a): 白色固体, 产率 $84 \%$. m.p. $137 \sim 139{ }^{\circ} \mathrm{C} ;{ }^{1} \mathrm{H}$ NMR (600 $\left.\mathrm{MHz}, \mathrm{CDCl}_{3}\right) \delta$ : $9.18(\mathrm{~s}, 1 \mathrm{H}), 7.96(\mathrm{~s}, 1 \mathrm{H}), 7.36 \sim 7.33(\mathrm{~m}$, $2 \mathrm{H}), 7.32 \sim 7.29(\mathrm{~m}, 3 \mathrm{H}), 6.83(\mathrm{~s}, 1 \mathrm{H}), 6.74(\mathrm{~s}, 1 \mathrm{H}), 5.15$ (s, 1H), $3.83(\mathrm{~s}, 3 \mathrm{H}) ;{ }^{13} \mathrm{C}$ NMR $\left(150 \mathrm{MHz}, \mathrm{CDCl}_{3}\right) \delta$ :
$161.62,143.44,138.71,128.65,128.56,127.60,123.36$, 122.75, 122.21, 115.48, 51.65, 51.14; IR (film) v: 3316, 2954, 1685, 1438, 1211, 1106, 994, 766, $636 \mathrm{~cm}^{-1}$; HR-MS-ESI calcd for $\mathrm{C}_{14} \mathrm{H}_{14} \mathrm{ClN}_{2} \mathrm{O}_{3}[\mathrm{M}+\mathrm{H}]^{+}$293.0687, found 293.0690.

4-(2-氯-2-肜基-1-(对甲苯基)乙基)-吡咯-2-甲酸甲 酯(3b): 白色固体，产率 $88 \%$. m.p. $133 \sim 135{ }^{\circ} \mathrm{C} ;{ }^{1} \mathrm{H}$ NMR (600 MHz, $\left.\mathrm{CDCl}_{3}\right) \delta$ : $9.39(\mathrm{~s}, 1 \mathrm{H}), 8.63(\mathrm{~s}, 1 \mathrm{H}), 7.18$ (d, $J=8.1 \mathrm{~Hz}, 2 \mathrm{H}), 7.14(\mathrm{~d}, J=7.9 \mathrm{~Hz}, 2 \mathrm{H}), 6.81(\mathrm{~s}, 1 \mathrm{H})$, $6.67(\mathrm{~s}, 1 \mathrm{H}), 5.09(\mathrm{~s}, 1 \mathrm{H}), 3.82(\mathrm{~s}, 3 \mathrm{H}), 2.33(\mathrm{~s}, 3 \mathrm{H}) ;{ }^{13} \mathrm{C}$ NMR $\left(150 \mathrm{MHz}, \mathrm{CDCl}_{3}\right) \delta: 161.97,143.60,137.33$, $135.75,129.38,128.43,123.56,122.53,122.47,115.68$, 51.73, 50.87, 21.11; IR (film) v: 3297, 1684, 1444, 1398, 1272, 1083, 988, 760, $658 \mathrm{~cm}^{-1}$; HR-MS-ESI calcd for $\mathrm{C}_{15} \mathrm{H}_{16} \mathrm{ClN}_{2} \mathrm{O}_{3}[\mathrm{M}+\mathrm{H}]^{+}$307.0844, found 307.0841.

4-(2-氯-2-肜基-1-(2-氟苯基)乙基)-吡咯-2-甲酸甲 酯(3c): 白色固体，产率 $85 \%$. m.p. $139 \sim 141{ }^{\circ} \mathrm{C} ;{ }^{1} \mathrm{H}$ NMR (600 MHz, $\left.\mathrm{CDCl}_{3}\right) \delta: 9.21(\mathrm{~s}, 1 \mathrm{H}), 8.05(\mathrm{~s}, 1 \mathrm{H})$, $7.30 \sim 7.27(\mathrm{~m}, 2 \mathrm{H}), 7.13 \sim 7.05(\mathrm{~m}, 2 \mathrm{H}), 6.83(\mathrm{~s}, 1 \mathrm{H})$, $6.77(\mathrm{~s}, 1 \mathrm{H}), 5.43(\mathrm{~s}, 1 \mathrm{H}), 3.83(\mathrm{~s}, 3 \mathrm{H}) ;{ }^{13} \mathrm{C}$ NMR $(150$ $\left.\mathrm{MHz}, \mathrm{CDCl}_{3}\right) \delta: 161.59,160.41$ (d, $\left.J=247.6 \mathrm{~Hz}\right), 142.24$, 129.90 (d, $J=3.2 \mathrm{~Hz}), 129.38$ (d, $J=8.3 \mathrm{~Hz}), 126.26$ (d, $J=14.0 \mathrm{~Hz}), 124.22(\mathrm{~d}, J=3.6 \mathrm{~Hz}), 122.85,122.19$, $122.17,115.57$ (d, $J=21.9 \mathrm{~Hz}), 115.38,51.68,44.23$ (d, $J=3.9 \mathrm{~Hz}$ ); IR (film) v: 3297, 1678, 1440, 1391, 1232, 1110, 995, 973, 768, $635 \mathrm{~cm}^{-1}$; HR-MS-ESI calcd for $\mathrm{C}_{14} \mathrm{H}_{13} \mathrm{ClFN}_{2} \mathrm{O}_{3}[\mathrm{M}+\mathrm{H}]^{+}$311.0593, found 311.0593.

4-(2-氯-2-肜基-1-(4-氟苯基)乙基)-吡咯-2-甲酸甲 酯(3d): 白色固体，产率 $83 \%$. m.p. $116 \sim 118{ }^{\circ} \mathrm{C} ;{ }^{1} \mathrm{H}$ NMR (600 MHz, $\left.\mathrm{CDCl}_{3}\right) \delta$ : $9.27(\mathrm{~s}, 1 \mathrm{H}), 8.19(\mathrm{~s}, 1 \mathrm{H})$, $7.29 \sim 7.27(\mathrm{~m}, 2 \mathrm{H}), 7.05 \sim 7.02(\mathrm{~m}, 2 \mathrm{H}), 6.81(\mathrm{~s}, 1 \mathrm{H})$, $6.73(\mathrm{~s}, 1 \mathrm{H}), 5.13(\mathrm{~s}, 1 \mathrm{H}), 3.84(\mathrm{~s}, 3 \mathrm{H}) ;{ }^{13} \mathrm{C}$ NMR $(150$ $\left.\mathrm{MHz}, \mathrm{CDCl}_{3}\right) \delta: 162.15(\mathrm{~d}, J=246.5 \mathrm{~Hz}), 161.66,143.22$, $134.43(\mathrm{~d}, J=3.2 \mathrm{~Hz}), 130.17(\mathrm{~d}, J=8.1 \mathrm{~Hz}), 123.20$, $122.79,122.14,115.54$ (d, $J=21.5 \mathrm{~Hz}), 115.34,51.74$, 50.36; IR (film) v: 3291, 1689, 1509, 1438, 1388, 1205, $1109,989,755,696 \mathrm{~cm}^{-1}$; HR-MS-ESI calcd for $\mathrm{C}_{14} \mathrm{H}_{13} \mathrm{Cl}-$ $\mathrm{FN}_{2} \mathrm{O}_{3}[\mathrm{M}+\mathrm{H}]^{+}$311.0593, found 311.0593 .

4-(2-氯-2-肜基-1-(4-氯苯基)乙基)-吡咯-2-甲酸甲 酯(3e): 淡黄色固体，产率 85\%. m.p. $104 \sim 106{ }^{\circ} \mathrm{C} ;{ }^{1} \mathrm{H}$ NMR (600 MHz, CDCl $) \delta: 9.42(\mathrm{~s}, 1 \mathrm{H}), 8.62(\mathrm{~s}, 1 \mathrm{H}), 7.31$ (d, $J=8.5 \mathrm{~Hz}, 2 \mathrm{H}), 7.23(\mathrm{~d}, J=8.4 \mathrm{~Hz}, 2 \mathrm{H}), 6.80(\mathrm{~s}, 1 \mathrm{H})$, 6.69 (s, 1H), $5.11(\mathrm{~s}, 1 \mathrm{H}), 3.83(\mathrm{~s}, 3 \mathrm{H}) ;{ }^{13} \mathrm{C}$ NMR $(150$ $\left.\mathrm{MHz}, \mathrm{CDCl}_{3}\right) \delta: 161.84,142.92,137.23,133.51,129.90$, $128.82,122.87,122.71,122.31,115.46,51.81,50.50$; IR 
(film) $v: 3290,1686,1498,1397,1268,1081,1001,765$, $705 \mathrm{~cm}^{-1}$; HR-MS-ESI calcd for $\mathrm{C}_{14} \mathrm{H}_{13} \mathrm{Cl}_{2} \mathrm{~N}_{2} \mathrm{O}_{3}[\mathrm{M}+\mathrm{H}]^{+}$ 327.0298 , found 327.0291 .

4-(2-氯-2-肜基-1-(4-溴苯基)乙基)-吡咯-2-甲酸甲 酯(3f), 白色固体, 产率 87\%. m.p. 106 108 ${ }^{\circ} \mathrm{C} ;{ }^{1} \mathrm{H}$ NMR (600 MHz, $\left.\mathrm{CDCl}_{3}\right) \delta$ : $9.40(\mathrm{~s}, 1 \mathrm{H}), 8.58(\mathrm{~s}, 1 \mathrm{H}), 7.46$ (d, $J=8.5 \mathrm{~Hz}, 2 \mathrm{H}), 7.17$ (d, $J=8.4 \mathrm{~Hz}, 2 \mathrm{H}), 6.79$ (s, 1H), 6.69 (s, 1H), 5.09 (s, 1H), 3.83 (s, 3H); ${ }^{13} \mathrm{C}$ NMR (150 $\left.\mathrm{MHz}, \mathrm{CDCl}_{3}\right) \delta: 161.80,142.82,137.77,131.78,130.26$, 122.78, 122.74, 122.28, 121.66, 115.43, 51.81, 50.57; IR (film) $v$ : 3273, 1690, 1486, 1436, 1388, 1205, 1109, 989, 755, $696 \mathrm{~cm}^{-1}$; HR-MS-ESI calcd for $\mathrm{C}_{14} \mathrm{H}_{13} \mathrm{BrClN}_{2} \mathrm{O}_{3}$ $[\mathrm{M}+\mathrm{H}]^{+}$370.9793, found 370.9791 .

4-(2-氯-2-肜基-1-(4-甲氧基苯基)乙基)-吡咯-2-甲 酸甲酯 $(\mathbf{3 g})$ : 淡黄色固体, 产率 88\%. m.p. 94 96 ${ }^{\circ} \mathrm{C}$; ${ }^{1} \mathrm{H}$ NMR (600 MHz, $\left.\mathrm{CDCl}_{3}\right) \delta: 9.49(\mathrm{~s}, 1 \mathrm{H}), 8.94$ (s, $1 \mathrm{H}), 7.21(\mathrm{~d}, J=8.6 \mathrm{~Hz}, 2 \mathrm{H}), 6.86(\mathrm{~d}, J=8.6 \mathrm{~Hz}, 2 \mathrm{H})$, $6.80(\mathrm{~s}, 1 \mathrm{H}), 6.66(\mathrm{~s}, 1 \mathrm{H}), 5.08(\mathrm{~s}, 1 \mathrm{H}), 3.81(\mathrm{~s}, 3 \mathrm{H}), 3.79$ (s, 3H); ${ }^{13} \mathrm{C}$ NMR $\left(150 \mathrm{MHz}, \mathrm{CDCl}_{3}\right) \delta: 162.00,158.94$, $143.62,130.79,129.67,123.70,122.50,122.49,115.64$, 114.03, 55.32, 51.77, 50.41; IR (film) $v: 3255,1694,1487$, $1444,1389,1109,998,755,702 \mathrm{~cm}^{-1}$; HR-MS-ESI $z$ calcd for $\mathrm{C}_{15} \mathrm{H}_{16} \mathrm{ClN}_{2} \mathrm{O}_{4}[\mathrm{M}+\mathrm{H}]^{+}$323.0793, found 323.0791 .

4-(2-氯-2-肜基-1-(3-溴苯基)乙基)-吡咯-2-甲酸甲 酯(3h): 淡黄色固体, 产率 80\%. m.p. 92 94 ${ }^{\circ} \mathrm{C} ;{ }^{1} \mathrm{H}$ NMR (600 MHz, $\left.\mathrm{CDCl}_{3}\right) \delta$ : 9.45 (brs, 1H), $8.72(\mathrm{~s}, 1 \mathrm{H})$, $7.43 \sim 7.41(\mathrm{~m}, 2 \mathrm{H}), 7.24 \sim 7.19(\mathrm{~m}, 2 \mathrm{H}), 6.81(\mathrm{~s}, 1 \mathrm{H})$, $6.71(\mathrm{~s}, 1 \mathrm{H}), 5.10(\mathrm{~s}, 1 \mathrm{H}), 3.83(\mathrm{~s}, 3 \mathrm{H}) ;{ }^{13} \mathrm{C}$ NMR $(150$ $\left.\mathrm{MHz}, \mathrm{CDCl}_{3}\right) \delta: 161.89,142.66,141.09,131.57,130.76$, $130.18,127.20,122.75,122.70,122.57,122.40,115.52$, 51.82, 50.73; IR (film) v: 3272, 1690, 1487, 1435, 1388, 1200, 1115, 989, 755, $700 \mathrm{~cm}^{-1}$; HR-MS-ESI calcd for $\mathrm{C}_{14} \mathrm{H}_{13} \mathrm{BrClN}_{2} \mathrm{O}_{3}[\mathrm{M}+\mathrm{H}]^{+}$370.9793, found 370.9791.

4-(2-氯-2-肜基-1-(3,4-二甲氧基苯基)乙基)-吡咯-2甲酸甲酯(3i): 淡黄色固体，产率 $61 \%$. m.p. 154 $156{ }^{\circ} \mathrm{C} ;{ }^{1} \mathrm{H}$ NMR $\left(600 \mathrm{MHz}, \mathrm{CDCl}_{3}\right) \delta: 8.85$ (brs, $\left.1 \mathrm{H}\right)$, $6.86 \sim 6.84(\mathrm{~m}, 2 \mathrm{H}), 6.78(\mathrm{dd}, J=8.2,2.1 \mathrm{~Hz}, 1 \mathrm{H}), 6.69(\mathrm{~d}$, $J=2.0 \mathrm{~Hz}, 1 \mathrm{H}), 6.14(\mathrm{t}, J=3.2 \mathrm{~Hz}, 1 \mathrm{H}), 4.97$ (dd, $J=12.4$, $7.5 \mathrm{~Hz}, 1 \mathrm{H}), 4.87(\mathrm{t}, J=7.6 \mathrm{~Hz}, 1 \mathrm{H}), 4.81(\mathrm{dd}, J=12.4,7.8$ $\mathrm{Hz}, 1 \mathrm{H}), 3.87$ (s, 3H), 3.84 (s, 3H), 3.81 (s, 3H); ${ }^{13} \mathrm{C}$ NMR $\left(150 \mathrm{MHz}, \mathrm{CDCl}_{3}\right) \delta: 161.32,149.61,149.12,134.65$, $129.09,122.88,119.94,115.83,111.68,110.89,108.04$, 78.76, 55.98, 55.97, 51.58, 42.58; IR (film) v: 3255, 1690, 1489, 1443, 1394, 1110, 997, 755, $700 \mathrm{~cm}^{-1}$; HR-MS-ESI calcd for $\mathrm{C}_{16} \mathrm{H}_{18} \mathrm{~N}_{2} \mathrm{O}_{6} \mathrm{Na}[\mathrm{M}+\mathrm{Na}]^{+}$357.1057, found
357.1064.

4-(2-氯-2-肜基-1-(2,4-二氯苯基)乙基)-吡咯-2-甲酸 甲酯 $(\mathbf{3 j})$ : 淡黄色粘稠物, 产率 $84 \% .{ }^{1} \mathrm{H}$ NMR $(600 \mathrm{MHz}$, $\left.\mathrm{CDCl}_{3}\right) \delta$ : 9.31 (brs, $\left.1 \mathrm{H}\right), 8.25(\mathrm{~s}, 1 \mathrm{H}), 7.42(\mathrm{~s}, 1 \mathrm{H}), 7.22 \sim$ $7.21(\mathrm{~m}, 2 \mathrm{H}), 6.78(\mathrm{~s}, 1 \mathrm{H}), 6.76(\mathrm{~s}, 1 \mathrm{H}), 5.51(\mathrm{~s}, 1 \mathrm{H}), 3.83$ (s, 3H); ${ }^{13} \mathrm{C}$ NMR $\left(150 \mathrm{MHz}, \mathrm{CDCl}_{3}\right) \delta: 161.51,141.80$, 135.59, 134.71, 134.07, 130.84, 129.54, 127.32, 123.08, 122.27, 121.77, 115.31, 51.73, 47.80; IR (film) v: 3290, 1688, 1502, 1400, 1268, 1079, 997, 765, $702 \mathrm{~cm}^{-1}$; HR-MS-ESI calcd for $\mathrm{C}_{14} \mathrm{H}_{12} \mathrm{Cl}_{3} \mathrm{~N}_{2} \mathrm{O}_{3}[\mathrm{M}+\mathrm{H}]^{+} 360.9908$, found 360.9904 .

4-(2-氯-2-肜基-1-苯基乙基)-吡咯-2-甲酸乙酯(3k): 白色固体, 产率 86\%. m.p. 93 95 ${ }^{\circ} \mathrm{C} ;{ }^{1} \mathrm{H}$ NMR $(600$ $\left.\mathrm{MHz}, \mathrm{CDCl}_{3}\right) \delta: 9.12(\mathrm{~s}, 1 \mathrm{H}), 7.42 \sim 7.32(\mathrm{~m}, 4 \mathrm{H}), 6.90(\mathrm{~s}$, $1 \mathrm{H}), 6.79(\mathrm{~s}, 1 \mathrm{H}), 5.07(\mathrm{~s}, 1 \mathrm{H}), 4.30(\mathrm{q}, J=7.1 \mathrm{~Hz}, 2 \mathrm{H})$, $1.34(\mathrm{t}, J=7.1 \mathrm{~Hz}, 3 \mathrm{H}) ;{ }^{13} \mathrm{C}$ NMR $\left(150 \mathrm{MHz}, \mathrm{CDCl}_{3}\right) \delta$ : 160.71, 135.86, 129.17, 128.26, 127.54, 124.02, 121.07, $120.75,119.79,113.70,60.68,35.43,14.38$; IR (film) $v$ : 3316, 2955, 1685, 1442, 1208, 1106, 995, 765, $640 \mathrm{~cm}^{-1}$; HR-MS-ESI calcd for $\mathrm{C}_{15} \mathrm{H}_{16} \mathrm{ClN}_{2} \mathrm{O}_{3}[\mathrm{M}+\mathrm{H}]^{+} 307.0884$, found 307.0887.

4-(2-氯-2-肜基-1-(3-溴苯基)乙基)-吡咯-2-甲酸乙 酯(31): 淡黄色固体, 产率 $82 \%$. m.p. $132 \sim 135{ }^{\circ} \mathrm{C} ;{ }^{1} \mathrm{H}$ NMR (600 MHz, $\left.\mathrm{CDCl}_{3}\right) \delta$ : 9.15 (brs, $\left.1 \mathrm{H}\right), 7.54 \sim 7.45$ (m, $2 \mathrm{H}), 7.33(\mathrm{~d}, J=7.7 \mathrm{~Hz}, 1 \mathrm{H}), 7.28 \sim 7.25(\mathrm{~m}, 2 \mathrm{H}), 6.92(\mathrm{~s}$, 1H), 6.78 (s, 1H), 5.04 (s, 1H), 4.31 (q, $J=7.1 \mathrm{~Hz}, 2 \mathrm{H})$, $1.35(\mathrm{t}, J=7.1 \mathrm{~Hz}, 3 \mathrm{H}) ;{ }^{13} \mathrm{C}$ NMR $\left(150 \mathrm{MHz}, \mathrm{CDCl}_{3}\right) \delta$ : $160.62,138.02,131.55,130.71,130.63,126.21,124.27$, $123.16,120.77,120.22,119.17,113.57,60.79,35.01$, 14.39; IR (film) v: 3271, 1691, 1487, 1430, 1388, 1202, $1114,989,757,701 \mathrm{~cm}^{-1}$; HR-MS-ESI calcd for $\mathrm{C}_{15} \mathrm{H}_{15} \mathrm{Br}-$ $\mathrm{ClN}_{2} \mathrm{O}_{3}[\mathrm{M}+\mathrm{H}]^{+}$384.9984, found 384.9982 .

\section{2 .4 化合物 $\mathbf{4 a} \sim \mathbf{4 m}$ 的合成}

方法 1: 将化合物 3a (2.92 g, $10 \mathrm{mmol}$ )溶于 $\mathrm{CH}_{3} \mathrm{OH}$ $(100 \mathrm{~mL})$ 中, 加入钯碳(钯的质量分数为 $10 \%, 2.1 \mathrm{~g}$ )和 2 $\mathrm{mL}$ 浓盐酸, 密封, 充入 $\mathrm{H}_{2}$ (常压), 室温反应 $12 \mathrm{~h}$, 过滤, 蒸除溶剂, 饱和 $\mathrm{Na}_{2} \mathrm{CO}_{3}$ 溶液碱化, EtOAc 萃取 3 次, 合 并有机相, 干燥, 蒸除溶剂得到粘稠物 $4 \mathrm{a} 1.93 \mathrm{~g}$, 产率 $79 \%$.

方法 2: 向溶有化合物 3a (1.16 g, $4 \mathrm{mmol})$ 的甲醇 $(25 \mathrm{~mL})$ 中加入 $\mathrm{NiCl}_{2} \cdot 6 \mathrm{H}_{2} \mathrm{O}(952 \mathrm{mg}, 4 \mathrm{mmol})$, 在冰浴条 件下搅拌 $5 \mathrm{~min}$, 加入 $\mathrm{NaBH}_{4}(1.82 \mathrm{~g}, 48 \mathrm{mmol})$, 冰浴反 应 $1 \mathrm{~h}$, 反应结束, 加入饱和 $\mathrm{NH}_{4} \mathrm{Cl}$ 溶液 $(25 \mathrm{~mL})$, 过滤, 蒸干溶剂, 添加 $\mathrm{HCl}(1 \mathrm{~mol} / \mathrm{L})$ 溶液调节 $\mathrm{pH}$ 至 4 5, 加 入 $\mathrm{CH}_{2} \mathrm{Cl}_{2}$ 溶液萃取两次, 保留水相, 添加 $\mathrm{NaOH}(1$ 
$\mathrm{mol} / \mathrm{L}$ ) 溶液调节 $\mathrm{pH}$ 至碱性, 使用 $\mathrm{CH}_{2} \mathrm{Cl}_{2}$ 萃取有机相两 次, 保留有机相, 无水 $\mathrm{Na}_{2} \mathrm{SO}_{4}$ 干燥过夜, 蒸干溶剂, 得 到粘稠物 4a $700 \mathrm{mg}$, 产率 71\%.

4-(2-氨基-1-苯基乙基)-吡咯-2-甲酸甲酯(4a): 淡黄 色粘稠物, 产率 79\%. ${ }^{1} \mathrm{H}$ NMR $\left(600 \mathrm{MHz}, \mathrm{CDCl}_{3}\right) \delta: 9.61$ (s, 1H), 7.30 (t, J=7.6 Hz, 2H), $7.26 \sim 7.20(\mathrm{~m}, 3 \mathrm{H}), 6.80$ $(\mathrm{s}, 1 \mathrm{H}), 6.73$ (s, 1H), 3.88 (t, $J=7.5 \mathrm{~Hz}, 1 \mathrm{H}), 3.81(\mathrm{~s}, 3 \mathrm{H})$, 3.23 (dd, $J=12.7,7.2 \mathrm{~Hz}, 1 \mathrm{H}), 3.18$ (dd, $J=12.7,7.8 \mathrm{~Hz}$, $1 \mathrm{H}) ;{ }^{13} \mathrm{C} \mathrm{NMR}\left(150 \mathrm{MHz}, \mathrm{CDCl}_{3}\right) \delta: 161.61,143.14$, $128.63,128.04,127.52,126.58,122.67,121.14,114.23$, 51.43, 47.84, 47.81; IR (film) v: 3025, 2948, 1692, 1436, 1209, 1105, 732, $699 \mathrm{~cm}^{-1}$; HR-MS-ESI calcd for $\mathrm{C}_{14} \mathrm{H}_{17} \mathrm{~N}_{2} \mathrm{O}_{2}[\mathrm{M}+\mathrm{H}]^{+}$245.1285, found 245.1287.

4-(2-氨基-1-(对甲苯基)乙基)-吡咯-2-甲酸甲酯 (4b): 淡黄色粘稠物, 产率 80\%. ${ }^{1} \mathrm{H}$ NMR $(600 \mathrm{MHz}$, $\left.\mathrm{CDCl}_{3}\right) \delta: 9.28(\mathrm{~s}, 1 \mathrm{H}), 7.16 \sim 7.09(\mathrm{~m}, 4 \mathrm{H}), 6.79(\mathrm{~s}, 1 \mathrm{H})$, $6.74(\mathrm{~s}, 1 \mathrm{H}), 3.84(\mathrm{t}, J=7.5 \mathrm{~Hz}, 1 \mathrm{H}), 3.82(\mathrm{~s}, 3 \mathrm{H}), 3.21$ $(\mathrm{dd}, J=12.6,7.1 \mathrm{~Hz}, 1 \mathrm{H}), 3.15$ (dd, $J=12.6,7.9 \mathrm{~Hz}, 1 \mathrm{H})$, $2.32(\mathrm{~s}, 3 \mathrm{H}) ;{ }^{13} \mathrm{C} \mathrm{NMR}\left(150 \mathrm{MHz}, \mathrm{CDCl}_{3}\right) \delta: 161.52$, $140.02,136.09$, 129.30, 127.86, 127.84, 122.60, 120.97, 114.14, 51.44, 47.84, 47.36, 21.01; IR (film) v: 2952, 1694, 1437, 1391, 1217, 1106, 977, $736 \mathrm{~cm}^{-1}$; HR-MS-ESI calcd for $\mathrm{C}_{15} \mathrm{H}_{19} \mathrm{~N}_{2} \mathrm{O}_{2}[\mathrm{M}+\mathrm{H}]^{+}$259.1441, found 259.1450.

4-(2-氨基-1-(2-氟苯基)乙基)-吡咯-2-甲酸甲酯(4c): 淡黄色粘稠物, 产率 $82 \% .{ }^{1} \mathrm{H} \mathrm{NMR}\left(600 \mathrm{MHz} \mathrm{CDCl}_{3}\right) \delta$ : $9.46(\mathrm{~s}, 1 \mathrm{H}), 7.24 \sim 7.18(\mathrm{~m}, 2 \mathrm{H}), 7.10 \sim 7.02(\mathrm{~m}, 2 \mathrm{H})$, $6.83(\mathrm{~s}, 1 \mathrm{H}), 6.81(\mathrm{~s}, 1 \mathrm{H}), 4.25(\mathrm{t}, J=7.5 \mathrm{~Hz}, 1 \mathrm{H}), 3.82(\mathrm{~s}$, $3 \mathrm{H}), 3.25 \sim 3.18(\mathrm{~m}, 2 \mathrm{H}) ;{ }^{13} \mathrm{C} \mathrm{NMR}\left(150 \mathrm{MHz}, \mathrm{CDCl}_{3}\right) \delta$ : $161.49,160.81$ (d, $J=245.1 \mathrm{~Hz}), 130.07$ (d, $J=14.4 \mathrm{~Hz})$, 128.88 (d, $J=4.5 \mathrm{~Hz}), 128.00$ (d, $J=8.3 \mathrm{~Hz}$ ), 126.20, 124.28 (d, $J=3.4 \mathrm{~Hz}), 122.70,121.25,115.56$ (d, $J=22.7$ $\mathrm{Hz}), 114.26,51.47,46.75,40.56$ (d, $J=1.1 \mathrm{~Hz}$ ); IR (film) $v: 3303,2950,1697,1487,1394,1201,1107,989,756$, $656 \mathrm{~cm}^{-1}$; HR-MS-ESI calcd for $\mathrm{C}_{14} \mathrm{H}_{16} \mathrm{FN}_{2} \mathrm{O}_{2}[\mathrm{M}+\mathrm{H}]^{+}$ 263.1190, found 263.1197 .

4-(2-氨基-1-(4-氟苯基)乙基)-吡咯-2-甲酸甲酯(4d): 淡黄色粘稠物, 产率 $84 \% .{ }^{1} \mathrm{H} \mathrm{NMR}\left(600 \mathrm{MHz}, \mathrm{CDCl}_{3}\right) \delta$ : 9.49 (s, 1H), 7.20 (dd, $J=8.5,5.4 \mathrm{~Hz}, 1 \mathrm{H}), 7.00$ (t, $J=8.7$ $\mathrm{Hz}, 1 \mathrm{H}), 6.77$ (s, 1H), 6.74 (s, 1H), 3.87 (t, J=7.4 Hz, 1H), $3.82(\mathrm{~s}, 3 \mathrm{H}), 3.22(\mathrm{dd}, J=12.6,7.2 \mathrm{~Hz}, 1 \mathrm{H}), 3.14(\mathrm{dd}, J=$ 12.6, 7.8 Hz, 1H); ${ }^{13} \mathrm{C}$ NMR (150 MHz, $\left.\mathrm{CDCl}_{3}\right) \delta: 161.55$ $(\mathrm{d}, J=244.6 \mathrm{~Hz}), 161.51,138.80(\mathrm{~d}, J=3.1 \mathrm{~Hz}), 129.40$ (d, $J=7.8 \mathrm{~Hz}), 127.34,122.77,120.98,115.38$ (d, $J=21.2$ $\mathrm{Hz}), 114.02$, 51.49, 47.82, 46.88; IR (film) v: 2952, 1702, 1566, 1389, 1211, 1094, 998, 764, $703 \mathrm{~cm}^{-1}$; HR-MS-ESI calcd for $\mathrm{C}_{14} \mathrm{H}_{16} \mathrm{FN}_{2} \mathrm{O}_{2}[\mathrm{M}+\mathrm{H}]^{+}$263.11901, found 263.1197.

4-(2-氨基-1-(4-氯苯基)乙基)-吡咯-2-甲酸甲酯(4e): 淡桔色粘稠物，产率 78\%. ${ }^{1} \mathrm{H}$ NMR $\left(600 \mathrm{MHz}, \mathrm{CDCl}_{3}\right) \delta$ : $9.50(\mathrm{~s}, 1 \mathrm{H}), 7.27(\mathrm{~d}, J=9.3 \mathrm{~Hz}, 2 \mathrm{H}), 7.18(\mathrm{~d}, J=8.1 \mathrm{~Hz}$, $2 \mathrm{H}), 6.76(\mathrm{~s}, 1 \mathrm{H}), 6.74(\mathrm{~s}, 1 \mathrm{H}), 3.89 \sim 3.86(\mathrm{~m}, 1 \mathrm{H}), 3.82$ (s, 3H), $3.22 \sim 3.16(\mathrm{~m}, 2 \mathrm{H}) ;{ }^{13} \mathrm{C} \mathrm{NMR}\left(150 \mathrm{MHz}, \mathrm{CDCl}_{3}\right)$ $\delta: 161.49,141.65,132.28,129.37,128.72,126.99,122.82$, 121.03, 114.02, 51.50, 47.61, 46.98; IR (film) v: 2949, 1692, 1572, 1436, 1391, 1211, 1107, 998, 764, $702 \mathrm{~cm}^{-1}$; HR-MS-ESI calcd for $\mathrm{C}_{14} \mathrm{H}_{16} \mathrm{ClN}_{2} \mathrm{O}_{2}[\mathrm{M}+\mathrm{H}]^{+}$279.0895, found 279.0895 .

4-(2-氨基-1-(4-溴苯基)乙基)-吡咯-2-甲酸甲酯(4f): 淡棕色粘稠物, 产率 70\%. ${ }^{1} \mathrm{H} \mathrm{NMR}\left(600 \mathrm{MHz}, \mathrm{CDCl}_{3}\right) \delta$ : 9.54 (s, 1H), 7.42 (d, $J=8.2 \mathrm{~Hz}, 2 \mathrm{H}), 7.12$ (d, $J=8.2 \mathrm{~Hz}$, $2 \mathrm{H}), 6.76(\mathrm{~s}, 1 \mathrm{H}), 6.64(\mathrm{~s}, 1 \mathrm{H}), 3.87 \sim 3.85(\mathrm{~m}, 1 \mathrm{H}), 3.82$ $(\mathrm{s}, 3 \mathrm{H}), 3.22 \sim 3.15(\mathrm{~m}, 2 \mathrm{H}) ;{ }^{13} \mathrm{C} \mathrm{NMR}\left(150 \mathrm{MHz}, \mathrm{CDCl}_{3}\right)$ $\delta: 161.49,142.19,131.67,129.77,128.63,126.59,122.81$, 121.03, 114.01, 51.50, 47.55, 47.09; IR (film) v: 2949, 1691, 1571, 1436, 1391, 1209, 1106, 1009, 732, $700 \mathrm{~cm}^{-1}$; HR-MS-ESI calcd for $\mathrm{C}_{14} \mathrm{H}_{16} \mathrm{BrN}_{2} \mathrm{O}_{2}[\mathrm{M}+\mathrm{H}]^{+} 323.0390$, found 323.0391 .

4-(2-氨基-1-(4-甲氧基苯基)乙基)-吡咯-2-甲酸甲酯 (4g): 淡黄色粘稠物, 产率 85\%. ${ }^{1} \mathrm{H}$ NMR (600 MHz, $\left.\mathrm{CDCl}_{3}\right) \delta: 9.23(\mathrm{~s}, 1 \mathrm{H}), 7.16(\mathrm{~d}, J=8.6 \mathrm{~Hz}, 2 \mathrm{H}), 6.85$ (d, $J=8.6 \mathrm{~Hz}, 2 \mathrm{H}), 6.78(\mathrm{~s}, 1 \mathrm{H}), 6.75(\mathrm{~s}, 1 \mathrm{H}), 3.87(\mathrm{t}, J=7.5$ $\mathrm{Hz}, 1 \mathrm{H}), 3.81$ (s, 3H), 3.78 (s, 3H), 3.22 (dd, $J=12.6,7.2$ $\mathrm{Hz}, 1 \mathrm{H}), 3.14$ (dd, $J=12.6,7.9 \mathrm{~Hz}, 1 \mathrm{H}) ;{ }^{13} \mathrm{C}$ NMR $(150$ $\left.\mathrm{MHz}, \mathrm{CDCl}_{3}\right) \delta: 161.47,158.24,134.88,128.93,127.72$, 122.64, 120.98, 114.09, 113.99, 55.25, 51.45, 47.64, 46.38; IR (film) v: 2924, 1693, 1509, 1437, 1390, 1243, 1105, 998, 732, $700 \mathrm{~cm}^{-1}$; HR-MS-ESI calcd for $\mathrm{C}_{15} \mathrm{H}_{19} \mathrm{~N}_{2} \mathrm{O}_{3}$ $[\mathrm{M}+\mathrm{H}]^{+}$275.1390, found 275.1389.

4-(2-氨基-1-(3-溴苯基)乙基)-吡咯-2-甲酸甲酯(4h): 淡黄色粘稠物，产率 $69 \% .{ }^{1} \mathrm{H} \mathrm{NMR}\left(600 \mathrm{MHz}, \mathrm{CDCl}_{3}\right) \delta$ : $9.44(\mathrm{~s}, 1 \mathrm{H}), 7.38(\mathrm{~s}, 1 \mathrm{H}), 7.36 \sim 7.34(\mathrm{~m}, 1 \mathrm{H}), 7.18 \sim 7.17$ $(\mathrm{m}, 1 \mathrm{H}), 6.77(\mathrm{~s}, 1 \mathrm{H}), 6.76(\mathrm{~s}, 1 \mathrm{H}), 3.86(\mathrm{t}, J=7.4 \mathrm{~Hz}, 1 \mathrm{H})$, 3.83 (s, 3H), 3.22 (dd, $J=12.7,7.3 \mathrm{~Hz}, 1 \mathrm{H}), 3.16$ (dd, $J=$ 12.7, $7.7 \mathrm{~Hz}, 1 \mathrm{H}) ;{ }^{13} \mathrm{C} \mathrm{NMR}\left(150 \mathrm{MHz}, \mathrm{CDCl}_{3}\right) \delta: 161.46$, $145.62,131.05,130.18,129.72,126.69,122.89,122.73$, 121.00, 114.03, 51.50, 47.58, 47.39; IR (film) v: 2953, 1692, 1570, 1436, 1390, 1207, 1106, 1012, 733, $701 \mathrm{~cm}^{-1}$; HR-MS-ESI calcd for $\mathrm{C}_{14} \mathrm{H}_{16} \mathrm{BrN}_{2} \mathrm{O}_{2}[\mathrm{M}+\mathrm{H}]^{+}$323.0390, found 323.0394 .

4-(2-氨基-1-(3,4-二甲氧基苯基)乙基)-吡咯-2-甲酸 
甲酯(4i): 淡黄色粘稠物, 产率 $81 \% .{ }^{1} \mathrm{H}$ NMR (600 MHz, $\left.\mathrm{CDCl}_{3}\right) \delta: 10.11(\mathrm{~s}, 1 \mathrm{H}), 6.84(\mathrm{t}, J=6.0 \mathrm{~Hz}, 1 \mathrm{H}), 6.74(\mathrm{dd}$, $J=8.2,1.8 \mathrm{~Hz}, 1 \mathrm{H}), 6.69$ (d, $J=1.7 \mathrm{~Hz}, 1 \mathrm{H}), 5.96$ (d, $J=$ $3.3 \mathrm{~Hz}, 1 \mathrm{H}), 3.98$ (t, $J=6.0 \mathrm{~Hz}, 1 \mathrm{H}), 3.87$ (s, 3H), 3.83 (s, $3 \mathrm{H}), 3.82(\mathrm{~s}, 3 \mathrm{H}), 3.38 \sim 3.19(\mathrm{~m}, 2 \mathrm{H}) ;{ }^{13} \mathrm{C}$ NMR $(150$ $\left.\mathrm{MHz}, \mathrm{CDCl}_{3}\right) \delta: 161.59,149.18,148.14,139.85,133.06$, $121.35,120.22,115.50,111.34,111.24,108.24,55.93$, 55.90, 51.30, 47.02, 46.29; IR (film) v: 2922, 1693, 1510, 1437, 1391, 1250, 1104, 998, 732, $701 \mathrm{~cm}^{-1}$; HR-MS-ESI calcd for $\mathrm{C}_{16} \mathrm{H}_{21} \mathrm{~N}_{2} \mathrm{O}_{4}[\mathrm{M}+\mathrm{H}]^{+}$305.1496, found 305.1507 .

4-(2-氨基-1-(2,6-二氯苯基)乙基)-吡咯-2-甲酸甲酯 (4j): 淡黄色粘稠物, 产率 79\%. ${ }^{1} \mathrm{H}$ NMR $(600 \mathrm{MHz}$, $\left.\mathrm{CDCl}_{3}\right) \delta: 9.57$ (br s, 1H), $7.39(\mathrm{~s}, 1 \mathrm{H}), 7.19 \sim 7.18(\mathrm{~m}$, $2 \mathrm{H}), 6.79(\mathrm{~s}, 1 \mathrm{H}), 6.78(\mathrm{~s}, 1 \mathrm{H}), 4.43(\mathrm{t}, J=7.3 \mathrm{~Hz}, 1 \mathrm{H})$, $3.83(\mathrm{~s}, 3 \mathrm{H}), 3.22 \sim 3.15(\mathrm{~m}, 2 \mathrm{H}) ;{ }^{13} \mathrm{C} \mathrm{NMR}(150 \mathrm{MHz}$, $\left.\mathrm{CDCl}_{3}\right) \delta: 161.42,139.35,135.92,134.80,132.65,132.61$, $131.67,129.45,129.37,127.38,127.05,125.64,122.93$, 121.36, 114.11, 51.50, 46.72, 43.12; IR (film) v: 2949, 1693, 1571, 1433, 1389, 1210, 1106, 998, 765, $700 \mathrm{~cm}^{-1}$; HR-MS-ESI calcd for $\mathrm{C}_{14} \mathrm{H}_{15} \mathrm{Cl}_{2} \mathrm{~N}_{2} \mathrm{O}_{2}[\mathrm{M}+\mathrm{H}]^{+} 313.0505$, found 313.0515 .

4-(2-氨基-1-苯基乙基)-吡咯-2-甲酸乙酯(4k)：淡黄 色粘稠物, 产率 $83 \% .{ }^{1} \mathrm{H}$ NMR $\left(600 \mathrm{MHz} \mathrm{CDCl}_{3}\right) \delta$ : 10.18 (br s, 1H), 7.32 (t, $J=7.4 \mathrm{~Hz}, 2 \mathrm{H}), 7.25$ (t, $J=7.2$ $\mathrm{Hz}, 1 \mathrm{H}), 7.18$ (d, J=7.4 Hz, 2H), 6.83 (d, $J=3.6 \mathrm{~Hz}, 1 \mathrm{H})$, $5.95(\mathrm{~d}, J=3.6 \mathrm{~Hz}, 1 \mathrm{H}), 4.27(\mathrm{q}, J=7.1 \mathrm{~Hz}, 2 \mathrm{H}), 4.10 \sim$ $4.07(\mathrm{~m}, 1 \mathrm{H}), 3.36 \sim 3.27(\mathrm{~m}, 2 \mathrm{H}), 1.33$ (t, $J=7.1 \mathrm{~Hz}, 3 \mathrm{H})$; ${ }^{13} \mathrm{C} \mathrm{NMR}\left(150 \mathrm{MHz}, \mathrm{CDCl}_{3}\right) \delta: 161.29,140.52,139.10$, $128.82,128.12,127.20,121.91,115.34,108.29$, 60.10, 46.70, 46.33, 14.49; IR (film) v: 3024, 2950, 1690, 1434, 1209, 1101, 732, $701 \mathrm{~cm}^{-1}$; HR-MS-ESI calcd for $\mathrm{C}_{15} \mathrm{H}_{19} \mathrm{~N}_{2} \mathrm{O}_{2}[\mathrm{M}+\mathrm{H}]^{+}$259.1441, found 259.1442.

4-(2-氨基-1-(3-溴苯基)乙基)-吡咯-2-甲酸乙酯(4I): 淡黄色粘稠物, 产率 $71 \% .{ }^{1} \mathrm{H} \mathrm{NMR}\left(600 \mathrm{MHz}, \mathrm{CDCl}_{3}\right) \delta$ : 9.03 (brs, $1 \mathrm{H}), 7.38(\mathrm{~s}, 1 \mathrm{H}), 7.36 \sim 7.32(\mathrm{~m}, 1 \mathrm{H}), 7.19 \sim$ $7.18(\mathrm{~m}, 2 \mathrm{H}), 6.78(\mathrm{~s}, 1 \mathrm{H}), 6.76(\mathrm{~s}, 1 \mathrm{H}), 4.30(\mathrm{q}, J=7.1$ $\mathrm{Hz}, 2 \mathrm{H}), 3.86$ (t, $J=7.4 \mathrm{~Hz}, 1 \mathrm{H}), 3.37 \sim 2.97(\mathrm{~m}, 2 \mathrm{H}), 1.35$ $(\mathrm{t}, J=7.1 \mathrm{~Hz}, 3 \mathrm{H}) ;{ }^{13} \mathrm{C} \mathrm{NMR}\left(150 \mathrm{MHz}, \mathrm{CDCl}_{3}\right) \delta: 160.95$, $145.61,131.05,130.18,129.73,126.69,126.68,123.32$, $122.73,120.66,113.80,60.43,47.57,47.40,14.45$; IR (film) $v$ : 2954, 1692, 1572, 1437, 1389, 1207, 1104, 1008, $731,700 \mathrm{~cm}^{-1}$; HR-MS-ESI calcd for $\mathrm{C}_{15} \mathrm{H}_{18} \mathrm{BrN}_{2} \mathrm{O}_{2}[\mathrm{M}+$ $\mathrm{H}]^{+}$337.0549, found 337.0546 .
辅助材料(Supporting Information) 产物 $4 \mathrm{a} \sim 4 \mathrm{I}$ 的 ${ }^{1} \mathrm{H}$ $\mathrm{NMR}$ 和 ${ }^{13} \mathrm{C} \mathrm{NMR}$ 谱图. 这些材料可以免费从本刊网站 (http://sioc-journal.cn/)上下载.

\section{References}

[1] Zhang, Y.; Zhao, Y. F.; Tebben, A, J.; Sheriff, S.; Ruzanov, M.; Mark P. Fereshteh, M. P.; Fan, Y.; Lippy, J.; Swanson, J.; Ho, C. P.; Wautlet, B. S.; Rose, A.; Parrish, K.; Yang, Z.; Donnell, A. F.; Zhang, L. P.; Fink, B. E.; Vite, G. D.; Augustine-Rauch, K.; Fargnoli, J.; Robert M. Borzilleri, R. M. ACS Med. Chem. Lett. 2018, 9, 1117.

[2] Guzii, A. G.; Makarieva, T. N.; Denisenko, V. A.; Gerasimenko, A. V.; Udovenko, A. A.; Popov, R. S.; Dmitrenok, P. S.; Golotin, V. A.; Fedorov, S. N.; Grebnev, B. B.; Stonik, V. A. J. Nat. Prod. 2019, 82, 1704.

[3] Merour, J. Y.; Routier, S.; Suzenet, F.; Joseph, B. Tetrahedron 2013, $69,4767$.

[4] Wucherer-Plietker, M.; Merkul, E.; Müller, T. J. J.; Esdar, C.; Knöchel, T.; Heinrich, T.; Buchstaller, H. P.; Greiner, H.; Dorsch, D.; Finsinger, D.; Calderini, M.; Bruge, D.; Grädler, U. Bioorg. Med. Chem. Lett. 2016, 26, 3073.

[5] (a) Mérour, J. Y.; Buron, F.; Plé, K.; Bonnet, P.; Routier, S. Molecules 2014, 19, 19935.

(b) Han, Y.; Dong, W.; Guo, Q. Q.; Li, X. F.; Huang, L. J. Eur. J. Med. Chem. 2020, 203,112506.

(c) Motati, R. M.; Amaradhi, R.; Ganesh, T. Bioorg. Med. Chem. 2020, 28, 115830.

(d) Ma, H. L.; Yan, X. J.; Xiao, Y. M.; Yuan, D. K.; Zhang, Z. H.; Fu, B.; Yuan, H. Z. Chin. J. Org. Chem. 2016, 36, 158 (in Chinese). (麻红利, 间晓静, 肖玉梅, 袁德凯, 张振华, 傅滨, 袁会珠, 有 机化学, 2016, 36, 158.)

[6] Ganser, C.; Lauermann, E.; Maderer, A.; Stauder, T.; Kramb, J. P.; Plutizki, S.; Kindler, T.; Moehler, M.; Dannhardt, G. J. Med. Chem. 2012, 55, 9531.

[7] Liu, Y. A.; Jin, Q. H.; Zou, Y. F.; Ding, Q.; Yan, S. S.; Wang, Z C.; Hao, X. S.; Nguyen, B.; Zhang, X. Y.; Pan, J. F.; Mo, T. T.; Jacobsen, K.; Lam, T.; Wu, T. Y-H.; Petrassi, H. M.; Bursulaya, B.; DiDonato, M.; Gordon, W. P.; Liu, B.; Baaten, J.; Hill, R.; Nguyen-Tran, V.; Qiu, M. H.; Zhang, Y. Kamireddy, Q. A.; Espinola, S.; Deaton, L.; Ha, S.; Harb, G.; Jia, Y.; Li, J.; Shen, W. J.; Schumacher, A. M.; Colman, K.; Glynne, R.; Pan, S. F.; McNamara, P.; Laffitte, B.; Meeusen, S.; Molteni, V.; Loren, J. J. Med. Chem. 2020, 63, 2958.

[8] (a) Zhou, N. N.; Nowicka-Sans, B.; McAuliffe, B.; Ray, N.; Eggers, B.; Fang, H.; Fan, L.; Healy, M.; Langley, D. R.; Hwang, C.; Lataillade, M.; Hanna, G. J.; Krystal, M. J. Antimicrob. Chemother. 2014, 69, 573 .

(b) Chen, K.; Risatti, C.; Bultman, M.; Soumeillant, M.; Simpson, J.; Zheng, B.; Fanfair, D.; Mahoney, D.; Mudryk, B.; Fox, R. J.; Hsaio, Y.; Murugesan, S.; Conlon, D. A.; Buono, F. G.; Eastgate, M. D. J. Org. Chem. 2014, 79, 8757.

[9] (a) Jeanty, M.; Blu, J.; Suzenet, F.; Guillaumet, G. Org. Lett. 2009, 11,5142 .

(b) Simmons, B. J.; Hoffmann, M.; Champagne, P. A.; Picazo, E.; Yamakawa, K.; Morrill, K. L.; Houk, K. N.; Garg, N. K. J. Am. Chem. Soc. 2017, 139, 14833.

(c) Alekseyev, R. S.; Amirova, S. R.; Terenin, V. I. Chem. Heterocycl. Compd. 2017, 53, 196.

[10] Zhang, Z. X.; Yang, Z.; Meanwell, N. A.; Kadow, J. F.; Wang. T. J. Org. Chem. 2002, 67, 2345.

[11] Osano, M.; Jhaveri, D. P.; Wipf, P. Org. Lett. 2020, 22, 2215.

[12] (a) Bultman, M. S.; Fan, J. Y.; Fanfair, D.; Soltani, M.; Simpson, J.; Murugesan, S.; Soumeillant, M.; Chen, K.; Risatti, C.; La Cruz, T. E.; Buono, F. G.; Hung, V.; Schild, R. L.; Ivy, S.; Sweeney, J. T.; Conlon, D. A.; Eastgate, M. D. Org. Process Res. Dev. 2017, 21, 1131 . 
(b) Huang, L.; Cai, Y.; Zheng, C.; Dai, L. X.; You, S. L. Angew. Chem., Int. Ed. 2017, 56, 10545.

[13] (a) Demopoulosv, V. J. J. Heterocycl. Chem. 1988, 25, 635.

(b) Lancianesi, S.; Palmieri, A.; Petrinia, M. Adv. Synth. Catal. 2013, 355, 3285.

[14] (a) Tu, Z. J.; Raju, B. R.; Liou, T. R.; Kavala, V.; Kuo, C. W.; Jang, Y. C.; Shih, Y. H.; Wang, C. C.; Yao, C. F. Tetrahedron 2009, 65, 2436.

(b) An, L. T.; Zou, J. P.; Zhang, L. L.; Zhang, Y. Tetrahedron Lett. $\mathbf{2 0 0 7}, 48,4297$.

(c) Hua, Y. Z.; Han, X. W.; Huang, L. H.; Wang, M. C. Chin. J. Org. Chem. 2018, 38, 237 (in Chinese). (华远照, 韩兴旺, 黄利华, 王敏灿, 有机化学, 2018, 38, 237.)

[15] Meshram, H. M.; Kumar, D. A.; Reddy, B. C. Helv Chim. Acto 2009, 92, 1002.

[16] Nomiyama, S.; Ogura, T.; Ishida, H.; Aoki, K.; Tsuchimoto, T. $J$. Org. Chem. 2017, 82, 5178. 